\title{
Eventos Paleoclimáticos de El Niños, La Niñas e Neutros no Pacífico Tropical e de Precipitação no Sudoeste e Leste da Amazônia
}

\author{
Nathiel de Sousa Silva ${ }^{1}$ (D), José Maria Brabo Alves ${ }^{1}$ (D), Emerson Mariano da Silva ${ }^{1}$ (D), \\ Rafael Rocha Lima ${ }^{1}$ \\ ${ }^{1}$ Mestrado Profissional em Climatologia e Aplicações nos Países da CPLP e África, \\ Universidade Estadual do Ceará, Fortaleza, CE, Brasil.
}

Recebido em: 26 de Junho de 2020 - Aceito em: 7 de Julho de 2020

\begin{abstract}
Resumo
Estudos climáticos associados às variáveis físicas da atmosfera para identificação de padrões e suas variações necessitam de séries de dados longas para terem resultados mais confiáveis. Nem sempre essas séries com medidas instrumentais têm essa longevidade de observações, por isso aproximações por técnicas paleoclimáticas são importantes para inferir variabilidades relacionadas a esses processos físicos da natureza. Variações da superfície de temperatura da superfície do mar (TSM) que ocasionam diferenças de pressão a superfície entre o oeste e leste do oceano Pacífico, chamadas de Oscilação Sul, são características que estão associadas a eventos de alterações de TSM no Pacífico Tropical (El Ninõs, La Niñas e Neutros), que impactam climaticamente várias áreas do globo. Foram usados proxies de precipitação referentes às áreas no oeste e leste do Pacifico, 50-1955 DC, do Índice de Oscilação Sul e de precipitação de duas áreas no sudoeste (1800-2012 DC) e leste (1800-2012 DC) da Amazônia. A metodologia base foi o cálculo de escores $\mathrm{Z}$ usados como medidas de variabilidade dos proxies. Os principais resultados mostraram que os proxies de IOS indicaram eventos de El Niños (La Niñas) associados a períodos de climas globais mais quentes no período RWP e MWP e mais frias no período DACP e LIA. Para as áreas da sudoeste e leste da Amazônia os proxies mostraram que ocorreu uma maior variação da precipitação nesses últimos 200 anos no setor sudoeste da mesma.
\end{abstract}

Palavras-chave: ENOS, IOS, variação climática.

\section{Paleclimatic Events Of El Ninos, La Niñas And Neutrals And Of Precipitation In Southern Ans Eastern Of Amazônia}

\begin{abstract}
Climatic studies associated with the physical variables of the atmosphere to identify patterns and their variations require long data series to have more reliable results. These series with instrumental measures do not always have this longevity of observations, so approximations using paleoclimatic techniques are important to infer variability related to these physical processes in nature. Variations on the sea surface temperature (SST) that cause pressure differences on the surface between the west and east of the Pacific Ocean, called the South Oscillation, are characteristics that are associated with events of SST changes in the Tropical Pacific (E1 Ninõs, La Niñas and Neutros), which climatically impact various areas of the globe. Proxies of precipitation were used for the areas in the west and east of the Pacific, 50-1955 DC, the South Oscillation Index and the precipitation of two areas in the southwest (1800-2012 DC) and east (1800-2012 DC) of the Amazon. The base methodology was the calculation of $Z$ scores used as measures of variability of the proxies. The main results showed that the IOS proxies indicated El Niños (La Niñas) events associated with periods of warmer global climates in the RWP and MWP period and colder in the DACP and LIA period. For the southwest and eastern areas of the Amazon, the proxies showed that there was a major variation in precipitation over the past 200 years in the southwest sector of the same.
\end{abstract}

Keywords: ENSO, SOI, climatic variation.

Autor de correspondência: Nathiel de Sousa Silva, nathiel.silva@uece.br. 


\section{Introdução}

Para entender o clima presente de uma região, e relacioná-lo a sua projeção futura, é necessário se conhecer as características desse clima no passado, conceito de paleoclima. Esses estudos paleoclimáticos são ferramentas que possibilitam entender como o clima passado da Terra se comportou e associá-lo as suas condições presentes, e tentar projetar ações a futuros cenários climáticos análogos a esses climas do passado.

Estudos climáticos associados às variáveis físicas como temperatura, precipitação e outras, para identificação de seus padrões e variabilidades, necessitam de séries de dados longas para terem resultados mais confiáveis. Nem sempre essas séries com medidas instrumentais têm essa longevidade de observações, por isso aproximações por técnica paleoclimáticas são importantes para inferir aumentos ou diminuições dessas variáveis a esses processos físicos da natureza (Trouet et al., 2009, Conroy et al., 2010, McGragor et al., 2010).

A variabilidade climática nos trópicos, em particular a precipitação, é altamente impactada pelas variações térmicas temperatura da superfície do mar (TSM) no Oceano Pacífico Tropical que caracterizam o fenômeno El Niño Oscilação Sul (ENOS), sendo uma medida do ENOS o chamado Índice de Oscilação Sul - IOS (Trenberth e Caron, 2000). A Oscilação Sul (OS) é um fenômeno de interação oceano-atmosfera, definido principalmente pela resposta a essas variações de TSM, induzindo a um balanço de pressão e massa na atmosfera que compreende trocas entre os setores leste do Pacífico em Tahiti e Darwin no oeste da bacia.

Yan et al. (2011) analisaram um índice de IOS definido pela reconstrução de proxies de precipitação nos setores leste e oeste do oceano Pacífico. Nesse estudo, os autores mostraram as variações desse IOS nos últimos dois mil anos (50-2000 DC). Esse IOS reconstruído apresentou correlação significativa com reconstruções de irradiância solar e de temperaturas no hemisfério norte (Yan et al. (2011)).

Como séries históricas de variáveis meteorológicas longas são raras, os relatos existentes tornam-se pontuais e qualitativos, transmitindo poucas informações das condições normais e dificultando identificar suas causas e variabilidades. Dessa forma, indicadores quantitativos e representativos de paleoclimas têm o potencial de fornecer evidências para mudanças climáticas de grande escala (Oliveira et al., 2015). Com isso, pratica-se o uso da técnica indireta com uso de testemunhos ou proxys, a qual se baseia em informações biológicas, geológicas ou geofísicas que possibilitam reconstruir e obter estimativas fidedignas das condições climáticas reais ocorridas no passado.

A região Amazônica é um dos centros no globo de formação de vapor dágua e convecção atmosférica pro- funda, e tem um importante papel de circulação geral da atmosfera e no balanço de energia no globo terrestre (Wang e Fu, 2007 e Hilker et al., 2014). Segundo Rocha et al. (2017), essa região se comporta como fonte de umidade para a atmosfera, recebendo vapor d'água tanto do transporte de origem oceânica quanto da evapotranspiração produzida pela própria floresta tropical.

Os aspectos hidroclimatológicos da Amazônia tem apresentado períodos de secas e cheias que tem aumentados em partes da região a partir dos anos de 1960 (Lewis et al., 2011, Gloor et al., 2013, Marengo e Espinosa, 2016). Essa aparente amplificação no ciclo hidrológico da Amazônica tem sido atribuida a impactos naturais e antropogênicos incluindo a interação de impactos ambientais como o desmatamento (Khanna et al., 2017, IPCC, 2013; Nobre et al., 2016).

Vários estudos de proxies de precipitação desde a década de 1960 têm sido feitos ao redor do globo (Fritts 1966; Griffin et al., 2013, Paredes-Villanueva et al., 2016). Contudo, segundo Granato-Souza et al. (2018), ainda há uma grande dificuldade na identificação desses proxies nos trópicos devido à existência de poucas espécies de arvores nativas com crescimento de anéis anuais que podem claramente datar seus calendários anuais de formação.

Para Mattos (2017), os anéis de crescimento, em número, numa seção próxima ao solo podem ser usados para se determinar a idade da árvore. Ademais, é prudente adicionar um razoável número de anos para compensar o tempo que a planta jovem levou para atingir aquela altura. Esse ramo da ciência, designado dendrocronologia, possibilita segundo Schons et al. (2018), o estudo do crescimento das árvores e sua relação com variáveis ambientais.

Alguns estudos de cronologias de precipitação têm sido desenvolvidos no oeste da bacia Amazônica (Bräuning et al., 2009 e Paredes-Villanueva et al., 2016). López et al. (2017), apresentou um bem datado e documentado estudo de reconstrução de anéis de árvores de totais de precipitação para a estação chuvosa da nascente do Rio Madeira no sudoeste da Amazônia.

Granato-Souza et al. 2018 e López et al. (2017) analisaram dados de proxies de precipitação de anéis de árvores para o sudoeste (1786-2016) e leste da Amazônia. (1799-2012). Em López et al. (2017), a reconstrução de precipitação foi correlacionada com variáveis atmosféricas observadas que influenciam estação chuvosa. Uma delas, a circulação anticiclônica sobre latitude média da América do Sul, a qual inclinada na direção equatorial possibilita o transporte de massa de ar extratropical fria e relativamente seca que converge com o ar úmido e quente para formar convecção profunda e fortes chuvas neste setor da região do sul da Amazônia.

No estudo de Granato-Souza et al. (2018), a cronologia de reconstituicão de precipitaçao na área do Rio Paru de 1786 a 2016 mostrou-se significativamente correlacio- 
nada com as observações instrumentais de precipitação entre 1939 a 2016. Esse proxie de precipitação também se mostrou relacionado com as variações de TSM no Atlântico e especialmente no Pacífico tropical, semelhante ao padrão mostrado de correlação entre as TSMs com os dados instrumentais de chuva da Amazônia oriental.

O objetivo desse estudo foi analisar um proxie de precipitação anual que infere o IOS entre 50-2000 DC, mostrando suas variabilidades classificadas em três classes: IOS positivo, se maior que 0,5 ; IOS negativo se menor que $-0,5$ e IOS neutro se maior ou igual a $-0,5$ e menor ou igual 0,5 com intuito de associar essas variabilidades a eventos de La Niña, El Niño e condições neutras no Pacífico Tropical. Além disso, são investigadas os anos de secas e cheias no sudoeste e leste da Amazônia, entre o final dos anos 1700 até 2012. A diferença desse estudo está na evidência de se inferir fenômenos ENOS e condições neutras no Pacífico Tropical por essas estimativas de proxies de precipitação a nas regiões da Indonésia e Ilhas Galapagos entre 50-2000 DC e suas associações com clima global nos períodos de RWP, DACP, LIA.

\section{Materiais e Métodos}

Neste estudo são usados proxies de precipitação que definiram um ÍOS de áreas no Pacífico leste na região das Ilhas Galápagos $\left(0,41^{\circ} 00^{\prime} \mathrm{S}\right.$ e $\left.90^{\circ} 16^{\prime} \mathrm{W}\right)$ e oeste na região da Indonésia $\left(6^{\circ} 12^{\prime} \mathrm{S}\right.$ e $\left.106^{\circ} 11^{\prime} \mathrm{E}\right)$ - 50-1955 DC e de duas áreas na Amazônia. Uma no sudoeste (13 $59^{\prime} \mathrm{S}$ e $63^{\circ} 25^{\prime} \mathrm{W}$ e $13^{\circ} 59^{\prime} \mathrm{S}$ e $61^{\circ} 25^{\prime} \mathrm{W}$ e $13^{\circ} 59^{\prime} \mathrm{S}$ e $61^{\circ} 25^{\prime} \mathrm{W}$ e $17^{\circ} 35^{\prime} \mathrm{S}$ e $61^{\circ} 21^{\prime} \mathrm{W}$ e $17^{\circ} 59^{\prime} \mathrm{S}$ e $61^{\circ} 25^{\prime} \mathrm{W}-1800-2012$ AC) e outra no leste $\left(0,98^{\circ} 00^{\prime} \mathrm{S}\right.$ e $53^{\circ} 32^{\prime} \mathrm{W}-1800-2012$ DC), obtidos do National Centers for Environmental Information - National Oceanic and Atmosheric Admistration NOAA-EUA do projeto de estudos paleoclimáticos do NCDC (Fig. 1).

Para definir o IOS, foram usados proxies dede totais anuais de precipitação da região da Indonésia e de Galapagos. Esses proxies na Indonésia foram derivadas de uma reconstrução de salinidade baseada em foraminíferos planctônicos $\delta 180$ e a razão $\mathrm{Mg} / \mathrm{Ca}$. Os foraminíferos são protistas rizópodes unicelulares. São envoltos por uma carapaça, a qual pode ser biomineralizada (calcita, aragonita, sílica), orgânica (quitinosa) ou resultar da aglutinação de fragmentos minerais e/ou biogênicos. Estes protistas vivem majoritariamente em ambiente marinho, alguns em ambiente mixohalino e raros em água doce (Siemensma et al., 2017).

De acordo com Petró (2018), os foraminíferos são amplamente utilizados no estudo das alterações oceanográficas e climáticas e seu estudo fossilífero permite estimar a profundidade, temperatura e salinidade das águas superficiais e de fundo dos mares em que viveram. O $\delta 180$ da calcita foraminiferal $(\delta 180 \mathrm{O})$ reflete os efeitos combinados de temperatura de calcificação $(\mathrm{T})$ e isótopo

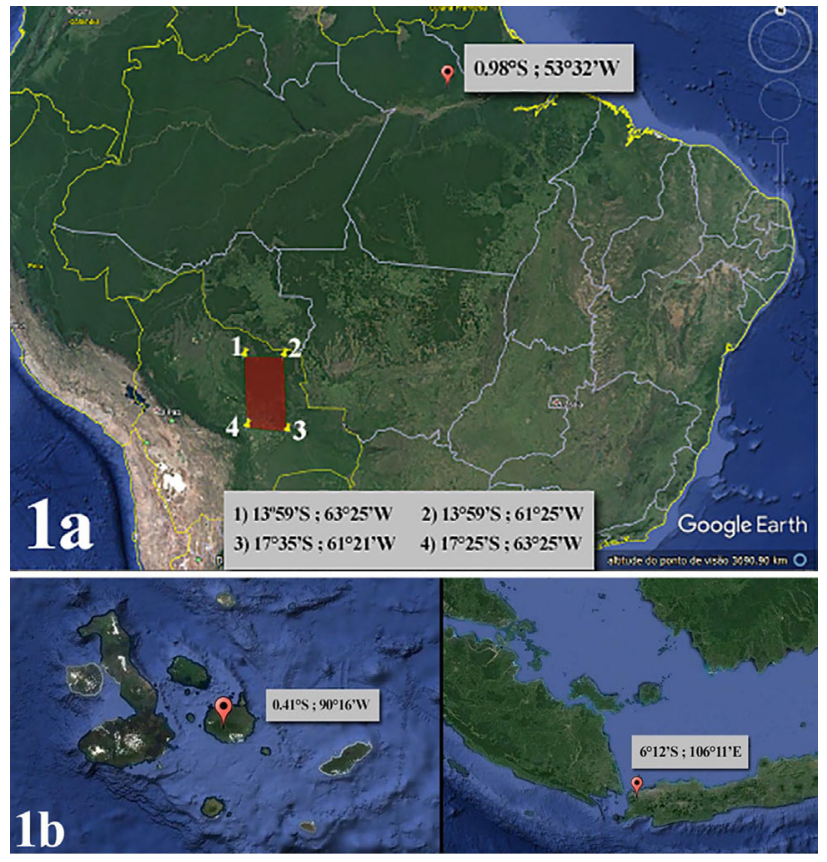

Fig. 1 - Localização das áreas de coleta dos proxies de precipitação. 1a) áreas no sudoeste e leste da Amazônia e 1b) áreas da Ilha Galápagos e Indonésia.

de oxigênio da água do mar ( $\delta 180 w)$, tendo o último variação em função da salinidade.

A relação $\mathrm{Mg} / \mathrm{Ca}$ nas conchas é principalmente dependente da temperatura (Oppo et al. 2010). Medindo ambos, $\delta 180$ c e a razão $\mathrm{Mg} / \mathrm{Ca}$, nas mesmas amostras, o componente de salinidade no sinal $\delta 180$ pode ser extraído. O componente de salinidade indica tendência de resfriamento causada pela precipitação das águas superficiais na piscina quente da região do Indico-Pacífico, sendo um indicador de precipitação (Yan et al. (2011)). Como esses proxies de IOS só estavam disponibilizados ate 1955, para o período de 1956 a 2019 foi usado um índice normalizado, médio anual, calculado com os dados mensais observados de da diferença pressão ao nível do mar (PNM) em Darwin e Thaiti disponíveis no site da NOAA (https://www.cpc.ncep.noaa. gov/data/indices/). Foram calculadas médias e desvio padrão anual dessa diferença e o índice normalizado ( $Z$ escore). O Z escore de uma amostra de dados refere-se é o número de desvios padrões acima ou abaixo da média da amostra.

Para Galápagos, os proxies de totais anuais de precipitação foram derivados de uma reconstrução de nível do lago, baseada em dados do tamanho dos grãos do núcleo de sedimentos de lago El Junco. Conforme sugerido em simulações de modelos hidro-climáticos, tamanho de grão de sedimentos do lago El Junco responde com sensibilidade às mudanças de precipitação associadas à circulação de Walker no Pacífico e ao El Niño (Conroy et al., 2008). 
Para os proxies de precipitação nos setores leste sudoeste da Amazônia foram usados uma compilação de anéis de ávores. No setor leste, os proxies são de uma espécie vegetal cientificamente chamada de Cedrela fissilis e Cedrela odorata, conhecida popularmente como Cedro nativa da região do Rio Paru, afluente do lado esquerdo do Rio Amazonas (Granato-Souza et al., 2018). Esses proxies são valores normalizados de anéis de árvores disponíveis somente para o período de fevereiro a novembro. Para o sudoeste da Amazônia, região da Amazônia Boliviana, os proxies são de totais anuais de precipitação derivados de um índice de anéis de árvores da espécie Entrolobium microchaete (López et al., 2017). Ambos os proxies estão disponibilizados no site da NOAA.

A metodologia de análise do estudo é descrita abaixo.

- Definição e identificação das classes de anos de IOS com o uso dos proxies de precipitação normalizados como: IOS (maior que 0,5 ; menor que $-0,5$ e Neutro se maior ou igual a $-0,5$ e menor ou igual 0,5 );

- Cálculo de percentuais de anos em cada uma das três classes em todo período de estudo 50-2019 DC, e nas chamadas eras: período romano quente (RWP-50-400 DC), período quente medieval (MWP-1000-1300 DC), período climático da idade Dark (DACP-500-900 DC) e período da pequena idade do Gelo (LIA- 1350-1850 DC);

- Analisar a variação dos IOSs nos períodos romano quente (RWP-50-400 DC), quente medieval (MWP1000-1300 DC), período climático da idade Dark (DACP-500-900 DC) e período da pequena idade do Gelo (LIA-1350-1850 DC). Nas regiões da Amazônia foi quantificado nas três classes de períodos chuvosos no leste e sudoeste da Amazônia, com os dados normalizados dos proxies usando os valores dos desvios anuais normalizados, como Secos (menor que -0,5), normal (maior ou igual a $-0,5$ e menor oi igual a 0,5 ) e chuvoso (maior que 0,5 ).

- Foi calculado como medida de variabilidade das séries de proxies um $Z$ escore médio das séries de precipitação para o setor sudoeste leste da Amazônia.

\section{Resultados e Discussão}

\section{1 - Análise da variação anual dos proxies de IOS (50- 1955 DC) e de IOS instrumental (1956-2019)}

A Fig. 2 mostra a variação interanual dos proxies e observações instrumentais do índice normalizado do IOS anual entre 50-2019 DC. Nota-se que nos períodos do RWP (50-400 DC) e MWP (1000-1300 DC), quando o clima global esteve mais quente, ocorreram valores de IOS negativos predominando, sugerindo a incidência de El Niños. Ao contrário, nos períodos DACP (500-900 DC) e
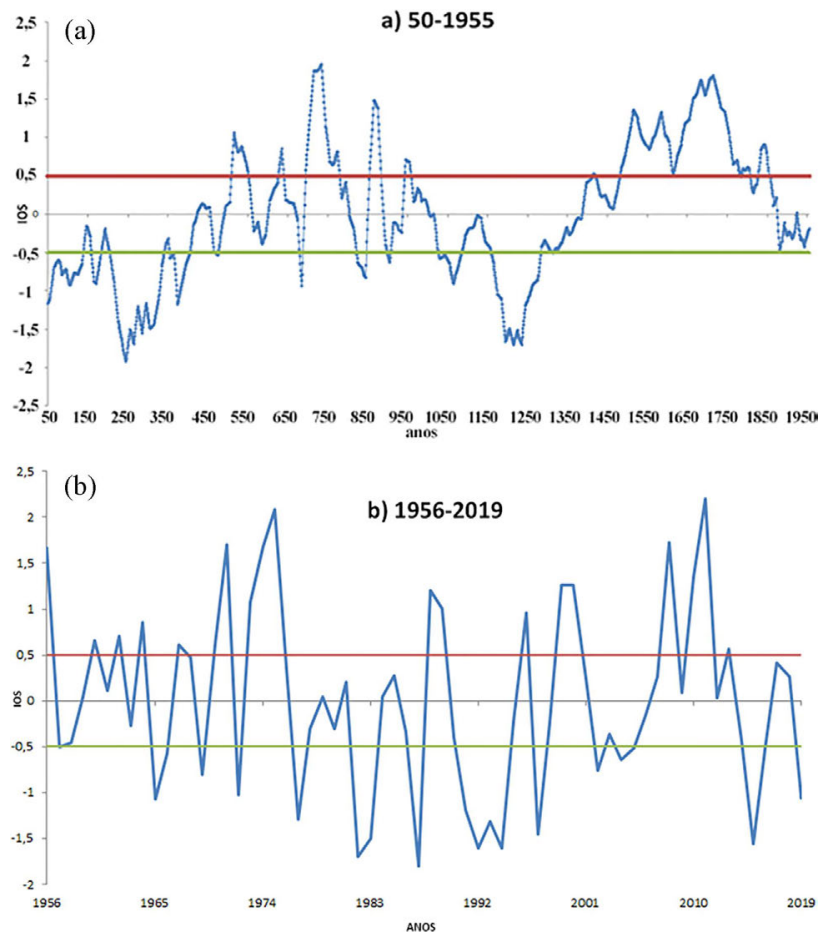

Fig. 2 - Variação anual do escore Z(normalizado) dos proxies e observações do índice do IOS (linha azul). a) proxies 50-1955 AC e b) observações instrumentais 1956-2019 AC. Linhas em vermelho e verde são limites que definiram IOSs associados a El Niños (valores acima da linha vermelha), La Niñas (abaixo da linha verde) e anos Neutros (entre as linhas verde e vermelha, inclusive).

LIA (1350-1850), quando o clima terrestre esteve mais frio, os valores dos proxies do IOS são predominantemente positivos, indicando a presença de La Niñas.

Essas inferências de períodos de El Niños e La Niñas pelos proxies em estudo são consistentes com os resultados mostrados em Yan et al. (2011), Bardet et al., 2000, Rein et al., 2005, os quais comentam que houveram evidências físicas no clima terrestre desses períodos, inferidos pelo radiação incidente em superfície, de maior (menor) incidência, em geral associados a anos de El Niños e La Niñas no sistema climático terrestre.

Para os períodos de LIA e DACP há vários estudos na literatura que inferem por proxies hidroclimáticos que condições de resfriamento no Pacifico Tropical e cheias em áreas costeiras da América do Sul estiveram presentes, indicativos de anos de La Ninãs (Rein et al., 2005).

$\mathrm{Na}$ Fig. 2a, outras características podem ser notadas em uma tendência linear positiva entre os anos 50-750 DC, decrescente de 750-1250 DC e crescente 1250-1955 DC. Nos dados instrumentais mais recentes de IOS podese ver uma variação interanual muito maior sem uma tendência definida com o índice oscilando a valores correspondentes a situações de El Niño, La Niña e neutras na bacia do Pacifico Tropical (Fig. 1b).

Os percentuais de IOS anuais estimados pelos proxies de precipitação e IOS observados a partir de 1955 , 
classificando em eventos de El Niño, La Niña e Neutros no Pacífico Tropical, mostraram entre 50-2019AC que ocorreram 29\% de anos com El Niños, 28\% de anos com La Niña e $43 \%$ dos anos com condição neutra no Pacífico Tropical. Para os períodos de RWP, DACP e LIA esses percentuais foram: RWP (0,0\% La Niñas, $85 \%$ El Niños e $15 \%$ Neutros), DACP (43\% La Nñas, $9 \%$ El Niños e $48 \%$ Neutros), MWP (0,0\% La Niñas, $61 \%$ El Niños, e 39\% Neutros), LIA (79\% La Niñas, 0,0\% El Niños e 21\% Neutros).

Os percentuais de anos com La Niñas, El Niños e Neutros inferidos pela classificação dos proxies do IOS para os respectivos períodos foram: RWP $(0,0 \%$ La Niñas, $85 \%$ El Niños e 15\% Neutros), DACP (43\% La Niñas, 9\% El Niños e 48\% Neutros), MWP (0,0\% La Niñas, $61 \%$ El Niños, e 39\% Neutros), LIA (79\% La Niñas, 0,0\% El Niños e 21\% Neutros).

\subsection{Análise da variação interanual dos proxies de precipitação no sudoeste e leste da Amazônia}

A Fig. 3 mostra a variação interanual dos escores $Z$ dos proxies e precipitação nas regiões sudoeste e leste da Amazônia em diferentes períodos.

$\mathrm{Na}$ Fig. 3a notou-se para todo o período uma maior variação entre os anos dos proxies de precipitação para o setor sudoeste da Amazônia. No setor leste da Amazônia esses escores de precipitação predominaram entre anos normais e chuvosos. No sudoeste também ocorreram secas consecutivas de três ou mais anos (1802-1806, 18241830), enquanto no leste da região essa característica foi evidente entre 1833-1836. Como os anos secos, os anos
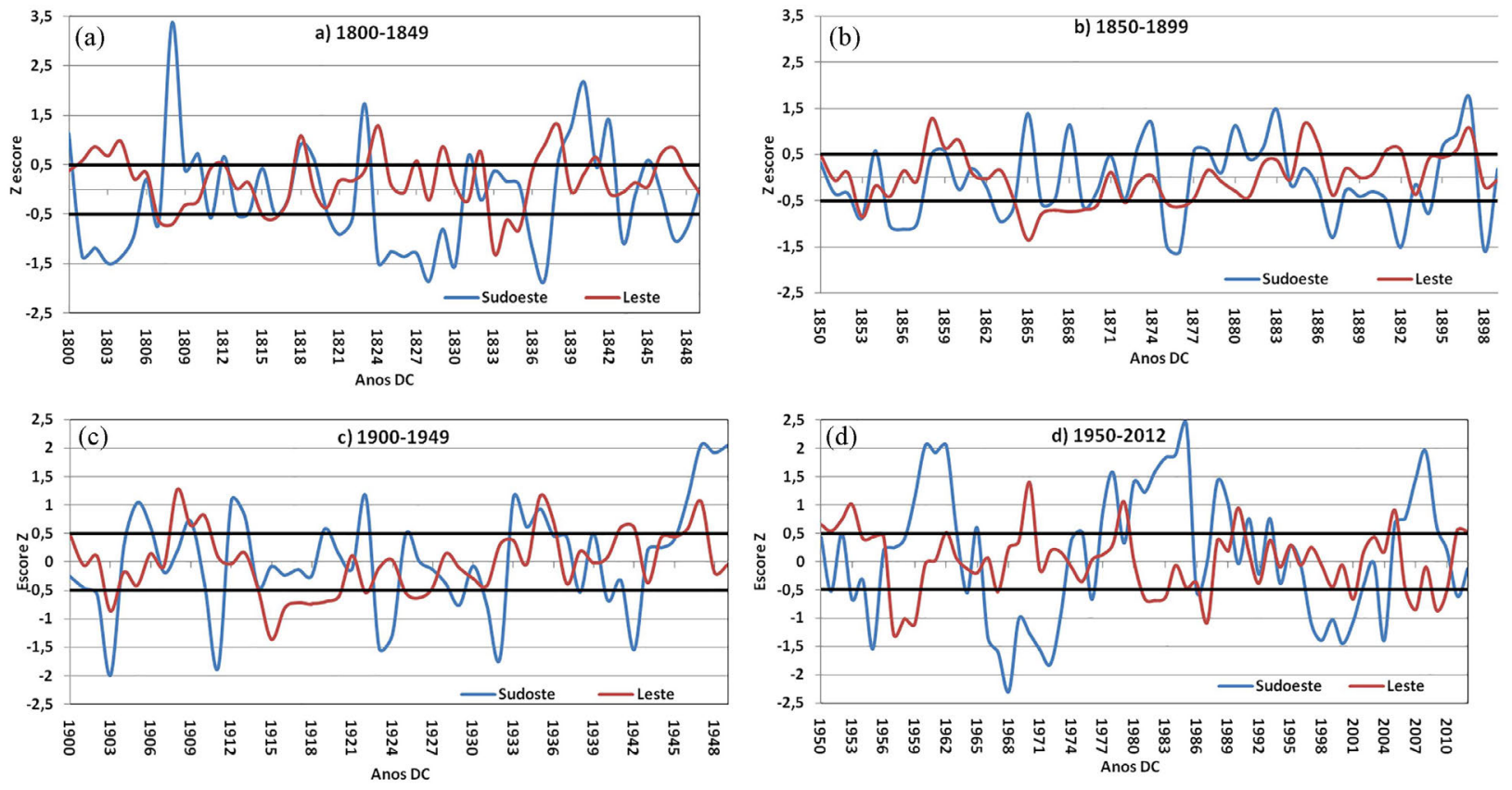

Figura 3 - a) Período de 1800-1849 DC. b) Período de 1850-1899 DC. c) Período de 1900-1949 DC. d) Período de 1950-2012 DC. As linhas pretas indicam o limite superior e inferior que definiram os anos classificados como de anos chuvosos, normais e secos. chuvosos também não foram homogêneos nesse século na Amazônia, ressaltam-se os anos de 1808, 1818-1820 e 1839-1842 no setor sudoeste e entre 1800-1806, 1818, $1824,1836-1839$ e 1847 no setor leste.

Essa diferença nesses proxies de precipitações nessas regiões se deve a circulação atmosférica e sistemas atmosféricos que causam chuvas nessas áreas da Amazônia (Marengo e Rogers, 2001; Hurley et al., 2015). Ressalta-se, que como esses proxies são em áreas pontuais da Amazônia a atuação de sistemas atmosféricos de escala intrasazonal, como linhas de instabilidades e convecção localizada, têm características de atuação diferentes nas regiões, o que deve ter causado essa variação de precipitação nessas áreas da Amazônia (Garreaud et al., 2009).

$\mathrm{Na}$ Fig. 3b observou-se uma maior amplitude interanual em magnitude dos proxies de precipitação para o setor sudoeste da Amazônia, como observado no período entre 1800-1849DC. No setor leste da Amazônia esses escores de precipitação predominaram, também como visto em 1800-1849 DC, entre anos normais e chuvosos. Nesse período no sudoeste ocorreram secas consecutivas maiores em magnitudes em valor do $\mathrm{Z}$ escore com períodos em anos menores do que ocorridos entre 1800-1849, observados por volta de 18561857 DC, 1863 DC, 1875-1876 DC, 1867 DC, 1892 DC e 1898 DC. No leste da bacia, ressalta-se um número de anos consecutivos de anos secos entre 18651870 DC. Os anos chuvosos nesse século de 1850-1899 DC ocorreram mais no setor sudoeste da bacia Amazônica, concentrando-se entre os anos de 1865-1868 DC e 1895-1897 DC. 
A Fig. 3c mostra uma maior amplitude interanual em magnitude dos proxies de precipitação para o setor sudoeste da Amazônia. No setor leste da Amazônia esses escores de precipitação novamente predominaram, entre anos normais e chuvosos. Nesse período no sudoeste ocorreram secas consecutivas maiores em magnitudes em valor do $\mathrm{Z}$ escore com períodos menores do que o s éculo anterior por volta de 1916 DC, 1924 DC, 1936 DC e 1945 DC. No leste da bacia, ressalta-se um número de anos consecutivos po $r$ volta dos anos de 1906 DC, 1912 DC, 1926 DC e 1932 DC. Os anos chuvosos nesse século de 1900-1949 DC ocorreram mais no setor sudoeste da Amazônia, sendo mais expressivos em Z escores em 1905 DC, 1909 DC, 1918 DC, 1925 DC, 1935 DC e partir de 1945 DC.

Na Fig. 3d, observa-se que, nesse período, o sudeste da bacia apresentou uma maior amplitude interanual, embora predominando anos classificados como normais de chuva. No sudoeste da Amazônia a amplitude foi mais marcada por períodos de anos consecutivos secos e chuvosos. Nesse período no sudoeste ocorreram secas consecutivas maiores em magnitudes em valor do $\mathrm{Z}$ escore entre 1968-1973DC, 1998-2001 DC, e chuvosos entre 1959-1963 DC entre 1982-1985 DC. No leste ressalta-se pelos proxies um período seco entre 1957-1959 DC e 2007 DC e 2010DC, e anos chuvosos em por volta dos anos de 1953 DC, 1970 DC, 1980 DC, 1989 DC e 2004 DC.

A Fig. 4 mostra a diferença dos proxies de precipitação para os períodos de 1800-1849 DC, 1850-1899 DC, 1900-1949 DC e 1950-2012 DC do número de anos classificados como anos secos, normais e chuvosos nos setores sudoeste e leste da Amazônia. Observa-se pelas duas figs. que no setor sudoeste a ocorrência de anos classificados nas três categorias, é mais próxima do que ocorreu para o setor leste com um predomínio de ocorrência de anos normais, com um número de anos bem maior que as outras duas categorias em todos os períodos analisados. Essa característica corrobora as análises das Figs. 2 que mostrou uma maior variação interanual da precipitação no oeste da Amazônia, embora o valor do $\mathrm{Z}$ escore médio para as duas regiões tenham sido muito próximos, foi $-0,05$ para o sudoeste e $-0,06$ para o setor leste da Amazônia.

\section{4 - Considerações Finais e Recomendações}

Em função dos resultados as principais considerações podem ser retiradas do estudo.

Os proxies de reconstituições do IOS de 50-1955 DC podem ser usados como indicadores do clima na região do Pacífico Tropical e inferirem as características da variabilidade térmica nessa bacia oceânica associada à ocorrência de eventos de La Ninas, El Niños e Neutros.

Foi possível identificar e relacionar as características climáticas evidenciadas nos estudos de paleoclimas em alguns períodos como RWP, MWP, DACP e LIA com as
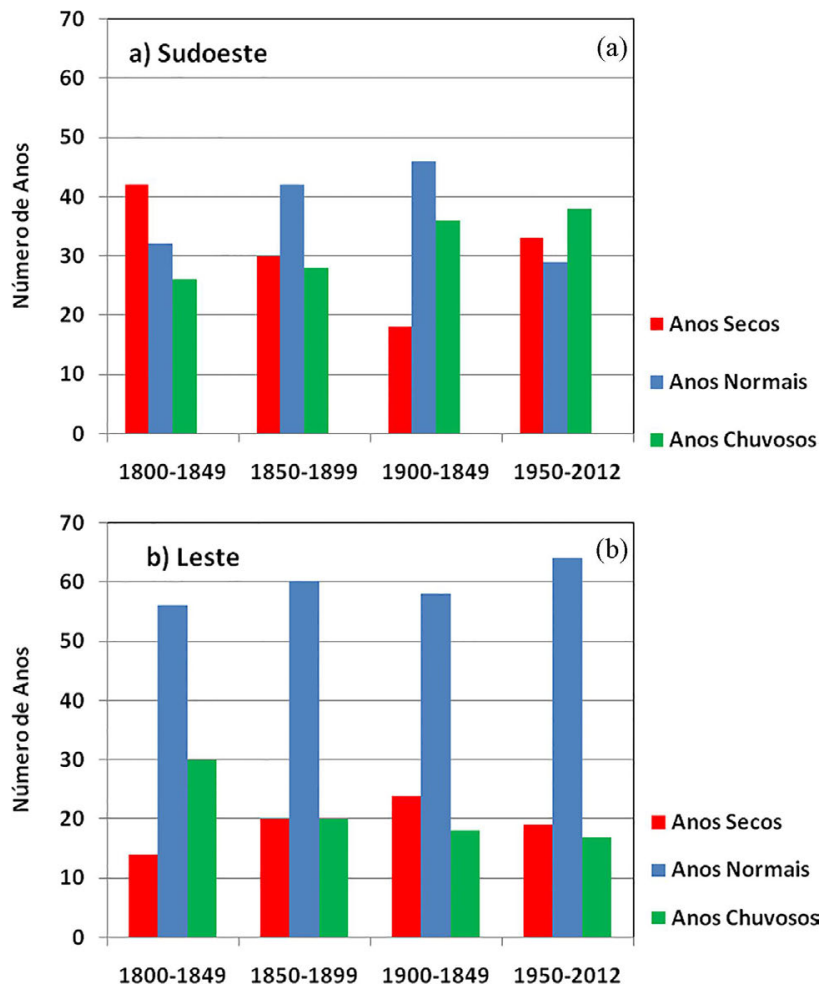

Fig. 4 - Número de anos classificados em anos secos, normais e chuvosos nos setores sudoeste e leste da Amazônia nos períodos de 1800-1849 DC, 1850-1899 DC, 1900-1949 DC e 1950-2012 DC. a) setor sudoeste e b) setor leste.

variabilidades dos proxies de IOS no Pacífico Tropical. Evidenciou-se pelos resultados que nos períodos mais quentes do RWP e MWP e frios do DACP e LIA, que a variabilidade interanual dos proxies de IOS indicou maior ocorrência de anos de El Niños e La Ninãs. Fisicamente associados às características da atmosfera, essas variações dos proxies do IOS nesses anos de El Niños e La Niñas foram respostas a variação da atividade convectiva da célula de Walker na bacia do Pacífico Tropical associadas às diferentes condições de TSMs na bacia (Yan et al., 2011).

Quanto à variação interanual dos proxies de precipitação nos setores sudoeste e leste da Amazônia nos períodos de estudo de 1800-1849 DC, 1850-1899 DC, 1900-1949 DC e 1950-1912 DC, os resultados mostraram que o setor sudoeste em todos os períodos de análise apresentou maior amplitude interanual entre anos secos, normais e chuvosos. Além disso, nessa região foram observados períodos de anos secos e chuvosos consecutivos muito mais frequentes e maiores em anos do que no setor leste. Este fato implica que nesses aproximadamente duzentos anos de análises a Amazônia boliviana teve uma maior variabilidade em seus mecanismos físicos que causam chuvas. Isto é, sugere uma grande irregularidade na incursão de ar frio associada às frentes frias oriundas de latitudes mais ao sul da América do Sul e do seu regime de 
monções (Gaurread e Wallace, 1998). Concomitantemente, na região do Rio Paru leste da Amazônia, os resultados sugeriram uma maior regularidade da atuação do principal sistema causador de precipitação que é a Zona de Convergência Intertropical (Shneyder et al., 2014).

Para estudos futuros sugere-se a utilização de outros tipos de proxies disponíveis para estimativa de precipitação sobre áreas da América do Sul para investigar e comparar as características das suas variações dos seus climas no passado com seus climas atuais, além de validar esses proxies com dados instrumentais, e relacionar esses proxies se possível com proxies de mecanismos físicos como a Oscilação Decadal do Pacífico (ODP) e Oscilação do Atlântico Norte (OAN).

\section{Referências}

BARD, E.; YIOU, R.; JOUZEL, G. Solar irradiance during the last 1200 years based on cosmogenic nuclides. Tellus B, v. 52, n. 3, p. 985-992, 2000.

BRAUNING, A.; VOLLAND, F.; BURCHARDT, I.; GANZHI, O. Climatic control of radial growth of Cedrelamontana in a humid mountain rainforest in southern Ecuador. Erdkunde, v. 63, n. 4, p. 337-345, 2009.

CONROY, J.L.; OVERPECK, J.T.; COLE, J.E.; SHANAHAN, T.M.; STEIINITZ-KANNAN, M. Holocene changes in eastern tropical Pacific climate inferred from a Galapagos lake sediment record. Quat. Sci. Rev, v. 27, n. 11-12, p. 1166-1180, 2008.

CONROY, J.L.; RESTREPO. A.; OVERPECK, J.T.; KANNAN, M.S. Unprecedented recent warming of surface temperatures in the eastern tropical Pacific. Ocean.Nature Geoscince, v. 2, n. 1, p. 46-50, 2009.

CONROY, J.L.; OVERPECK, J.T.; COLE, J.E. El Nino/Southern Oscillation and changes in the zonal gradient of tropical Pacific sea surface temperature over thelast1.2ka. Pages News, v. 18, n. 1, p. 32-34, 2010.

FRITTS, H.C. Growth rings of trees: their correlation with climate. Science, v. 25, n. 154T (3752), p. 973-979, 1966.

McGREGOR, S.; TIMMERMANN, A.; TIMMM, O. A unified proxy for ENSO and PDO variability since 1650. Climate of Past, v. 6, n.1, p. 1-17, 2010.

GARREAUD, R.D.; WALLACE, J.M. Wallace. Summertime incursions of midlatitude air into subtropical and tropical South America, Mon. Weather Rev., v. 126, n, 10, p. 2713-2733, 1998.

GARREAUD, R.D.; VUILLE, M..; CAMPAGNUCCI, R.; MARENGO, J. Present-day South American climate, Palaeogeogr. Palaeoclimatol. Palaeoecol., v. 281, n. 3-4, p. 180-195, 2009.

GLOOR, M.; BRIENEN, R.J.W.; GALBRAITH, D.; FELDPAUSCH, T.R.; SCHÖNGART, J.; GUYOT, J.L.; ESPINOZA, J.C.; LLOYD, J.; PHILLIPS, O.L. Intensification of the Amazon hydrological cycle over the last two decades. Geophysical Research Letter, v. 40, n. 9, p. 17291733, 2013.

GLOOR, M.; BARICHIVICH, J.; ZIV, G.; BRIENEN, R.; SCHÖNGART, J.; PEYLIN, P.; BARCANTE L.; CIN-
TRA, B.; FELDPAUSCH, T.; PHILLIPS, O.; BACKER, J. Recent Amazon climate as background for possible ongoing and future changes of Amazon humid forests. Global Biogeochemical Cycles, v. 29, n. 9, p. 1384-1399, 2015.

GRIFFIN, R.D.; WOODHOUSE, C.A.; MEKO, D.M.; STAHLE, D.W.; FAULSTICH, H.L.; CARRILLO, C.; TOUCHAN, R.; CASTRO, C.L.; LEAVITT, S.W. North American monsoon precipitation reconstructed from tree rings. Geophys Res Lett. v. 40, n. 5, p. 1-5, 2013.

HILKER, T.; LYAPUSTIN, A.I.; TUCKER, C.J.; HALL, F.G.; MYNENI, R.B.; WANG, Y.; BI, J.; MENDES, M.; SELLERS, Y. P.J. Vegetation dynamics and rainfall sensitivity of the Amazon. Proc Nat AcadSci, v. 111, n. 45, p. 1604116046, 2014.

KHANNA, J.; MEDVIGY, D.; FUEGLISTALER, S.; WALKO, R. Regional dry season climate changes due to three decades of Amazonian deforestation. Natural Climate Change, v. 7, n, 7, p. 200-204, 2017.

HURLEY, J.V.; VUILLE, M.; HARDY, D.R.; BURNS, S.J.; THOMPSON, L.G. Cold air incursions, $\delta 180$ variability, and monsoon dynamics associated with snow days at Quelccaya ice cap, Peru. J. Geophys. Res. Atmos., v. 120, n. 15, p. $7467-7487,2015$.

LEWIS, S.L.; BRANDO, P.M.; PHILLIPS, O.O.L; VANDER, H.G.M.F.; NEPSTAD, D. The 2010 Amazon drought. Science, v. 331, n. 6017, p. 554, 2011.

LÓPEZ, L.; STAHLE, D.; VILLALBA, R.; TORBENSON, M.; FENG, S.; COOK, E. Tree-ring reconstructed rainfall over the southern Amazon Basin. Geophysical Research Letter, v. 44, n. 14, p.7410-7418, 2017.

MARENGO, J.A.; ESPINOZA, J.C. Extreme seasonal droughts and floods in Amazonia: causes, trends and impacts. International Journal of Climatology, v. 36, n. 3, p. 10331050, 2016.

MARENGO, J.A.; ROGERS, J.C. Polar air outbreaks in the Americas: Assessments and impacts during modern and past climates, in Markgraf, V., ed., Interhemispheric Climate Linkages in the Americas. San Diego: Academic Press, p. 31-51, 2001.

NOBRE, C.A.; SAMPAIO, G.; BORNA, L.S.; CASTILLARUBIO, J.C.; SILVA, J.S.; CARDOSO, M. Land-use and climate change risks in the Amazon and the need of a novel sustainable development paradigm. Proc Nat AcadSci., v. 113, n. 39, p. 10759-10768, 2016.

OPPO, D.W.; ROSENTHAL, Y.; LINSLEY, B.K. 2,000-yearlong temperature and hydrology reconstructions from the Indo-Pacific warm pool. Nature, v. 460, n. 11, p. 11131116, 2009.

MATTOS, P.P. Identificação de Anéis Anuais de Crescimento e Estimativa da Idade e Incremento Anual em Diâmetro de Espécies Nativas do Pantanal da Nheconlândia-MS. Tese de Doutorado em Ciências Florestais, Setor de Ciências Agrárias, Universidade Federal do Paraná, Curitiba, p. 116, 1999.

OLIVEIRA, M.J.; BAPTISTA, G.M.M.; VECCHIA, F.V.; CARNEIRO, C.D.R.. História geológica e Ciência do Clima: métodos e origens do estudo dos ciclos climáticos na Terra. Terræ, v. 12, n. 1-2, p. 3-26, 2015.

PAREDES-VILLANUEVA， K.; LÓPEZ, L.; CERRILLO, R.M.N. Regional chronologies of Cedrelafissilis and 
Cedrelaangustifolia in three forest types and their relation to climate. Trees, v. 30, n.5, p. 1581-1593, 2016.

PETRÓ, S.M. Introdução ao Estudo dos Foraminíferos. Porto Alegre: IGEO/UFRGS, p. 12-13, 2018.

REIN, B.; LÜCKGE, A.; REINHARDT, L.; WOLF, A.; DULLO, W-C. El Nino variability off Peru during the last 20,000 years. Paleoceanography and Paleoclimatololy, v. 20, n. 44, p. 4003, 2005.

ROCHA, V.M.; CORREIA, F.W.; SILVA, P.R.T.; GOMES, W.B.; VERGASTA, L.A.; MOURA, R.G.; TRINDADE, M.S.P.; PEDROSA, A.L.; SILVA, J.J.S. Reciclagem de precipitação na Bacia Amazônica: o papel do transporte de umidade e da evapostranspiração da superfície. Revista Brasileira de Meteorologia, v. 32, n. 3, p. 387-398, 2017.

SCHONS, CRISTINE TAGLIAPIETRA et al. Dendrocronologia: princípios e aplicações. In: Anais da II Semana de Aperfeiçoamento em Engenharia Florestal da UFPR. Curitiba: CIFLOMA, capítulo 5, p. 12, 2018.

SHNEYDER, T.; BISCHOFF, T.; HAUG. G. H. Migrations and dynamics of the Intertropical Convergence Zone. Nature, v. 513, n. 7516, p. 45-53, 2014.

SIEMENSMA, F.; APOTHELOZ-PERRET-GENTIL, L.; HOLSMANN, M.; CLAUSS, S.; VÖLCKER, E.; PAWLOSKI, J. Taxonomic revision of freshwater foraminifera with the description of two new agglutinated species and genera. European Journal of Protistology, v. 60, p. 28-44, 2017.

GRANATO-SOUZA D., et al. Tree rings and rainfall in the equatorial Amazon. Climate Dynamics, v. 52, n. 3-4, p. 1857-1869, 2018.
TRENBERTH, K.E.; CARON, J.M. The Southern Oscillation revisited: Sea level pressures, surface temperatures, and precipitation. Journal of Climate, v.13, p. 4358-4365, 2000.

TROUET, V.; ESPER, J.; GRAHAM, N. E.; BAKER, A.; SCOURSE, J.D.; FRANK, D.C. Persistent positive north Atlantic oscillation mode dominated the medieval climate anomaly. Science, v. 324, n. 5923, p. 78-80, 2009.

VILLALBA, R.; COOK, E.R.; JACOBY, G.C.; D'ARIIGO, R.D.; VEBLEN, T.T.; JONES, P.D. Tree-ring based reconstructions of northern Patagonia precipitation since AD 1600. Holocene, v. 8, n. 6, p. 659-674, 1998.

YAN, H.; SUN, L.; OPPO, D.W.; WANG, Y.; LIU, Z.; XIE, Z.; LIU, X. CHENG, W. South China Sea hydrological changes and Pacific Walker Circulation variations over the last millennium. Nature Commun, v. 2, n. 293, p. 1-5, 2011.

YAN, H.; SUN, L.; WANG, H.; W, QIU, S.; YANG, C. Are record of the Southern Oscillation Index for the past 2,000 years from precipitation proxies. Nature Geoscience, v. 4, n. 9, p. 611-614, 2011.

WANG, H.; FU, R. The influence of Amazon rainfall on the Atlantic ITCZ through convectively coupled Kelvin waves. Journal of Climate, v. 20, n. 7, p. 1188-1201, 2007.

WANG, X.; EDWARDS, R.L.; AULER, A.S.; WANG, Y.; CRUZ, F.W.; DORALE, J.A.; CHIANG, H.W. Hydroclimatic changes across the Amazon lowlands over the past 45,000 years, Nature, v. 541, n. 7636, p. 204-207, 2017.

License information: This is an open-access article distributed under the terms of the Creative Commons Attribution License (type CC-BY), which permits unrestricted use, distribution and reproduction in any medium, provided the original article is properly cited. 